\title{
Prevalence, Risk Factors And Treatment Of The Most Common Gram-Negative Bacterial Infections In Liver Transplant Recipients: A Review
}

This article was published in the following Dove Press journal: Infection and Drug Resistance

\author{
Mojtaba Shafiekhani (iD) ${ }^{1,2}$ \\ Mahtabalsadat Mirjalili (iD ${ }^{\prime}$ \\ Afsaneh Vazin (iD) \\ 'Department of Clinical Pharmacy, \\ Faculty of Pharmacy, Shiraz University of \\ Medical Sciences, Shiraz, Iran; ${ }^{2}$ Shiraz \\ Organ Transplant Center, Abu-Ali Sina \\ Hospital, Shiraz University of Medical \\ Sciences, Shiraz, Iran
}

\begin{abstract}
Advances in surgical techniques and immunosuppressive agents have made solid organ transplant (Tx) an important strategy for treatment of end-stage organ failures. However, the incidence of infections following Tx due to Gram-negative pathogens is on the rise. These infections are associated with increased mortality and morbidity in patients following transplantation, including liver Tx. Thus, managing infections in liver Tx recipients is a big challenge, requiring prompt medical attention. Considering the important effect of Gramnegative bacterial infections on the outcomes of liver Tx recipients, the most prevalent Gramnegative pathogens including Klebsiella pneumoniae, Acinetobacter baumannii, Pseudomonas aeruginosa and Escherichia coli will be discussed in this review.
\end{abstract}

Keywords: liver transplantation, infection, Klebsiella pneumoniae, Acinetobacter baumannii, Pseudomonas aeruginosa, Escherichia coli

\section{Introduction}

Nowadays, solid organ transplant (Tx) is considered as an important strategy to treat many end-stage organ failures. ${ }^{1}$ Despite advances in surgical techniques, immunosuppressant drugs regimen, hospital care, and the identification methods of post-transplant complications, the bacterial infections are still the most important causes of patients' mortality and morbidity following solid organ $\mathrm{Tx} .{ }^{2}$ Liver transplant patients are more prone to post-transplant infections due to complication related to surgical method, since it has to penetrate into hepatobiliary system. ${ }^{3}$ Evidence has shown that bacterial, followed by viral and fungal infections are the most predominant infections following liver Tx, particularly during hospitalization. ${ }^{4}$

The incidence of bacterial infection and the pattern of pathogens sensitivity/ resistance are different from center to center, depending on different prophylactic protocols. For instance, $\mathrm{Li}$ et al reported that $14.1 \%$ of patients experienced bacterial infections within 3 months after $\mathrm{Tx}^{5}{ }^{5}$ while this rate was $30.2 \%$ in a 9-year study by Kim et al. ${ }^{6}$

A national prospective cohort study in 2018 was conducted to determine the incidence of infections in liver Tx recipients and reported that bacterial infections occurred in $31.7 \%$ of patients and the mortality rate caused by these infections was $6.4 \%$. The most common bacterial infections were sepsis $(16.1 \%)$, followed by urinary tract infections $(9.4 \%)$, pneumonia $(6.3 \%)$, and surgical site infections $(4.6 \%){ }^{7}$ 
In recent years, studies have shown that the Gramnegative microorganisms are more responsible for infections in liver $\mathrm{Tx}$ recipients in comparison with Gram-positive ones. ${ }^{8,9}$ Alberto Ferrarese et al reported that Enterobacteriaceae was responsible for $44.3 \%$ of hospital acquired infections within 1 month after liver Tx. In this regard, Escherichia coli, Klebsiella pneumoniae, and Proteus mirabilis were the most common pathogens responsible for infection. ${ }^{10}$ In a retrospective analysis of post-liver Tx infections, female gender, septic shock and lymphocyte counts below $300 / \mathrm{mm}^{3}$ were identified as the risk factors for mortality caused by Enterococcus faecium, Staphylococcus aureus, Klebsiella pneumoniae, Acinetobacter baumannii, Pseudomonas aeruginosa, and Enterobacter spp. ${ }^{11}$

It is clear that bacterial infection prophylaxis and treatment, as well as reducing infection-related complications, length of hospital stay, and total treatment costs are major concerns for liver Tx recipients. Therefore, the researchers will attempt to discuss the latest facts on epidemiology, risk factors, treatment options, and the impact of prophylactic strategies related to the most common Gram-negative bacterial infections in liver Tx recipients.

\section{Klebsiella pneumoniae (K. pneumoniae) Epidemiology}

Prevalence of $K$. pneumoniae infection in liver Tx recipients varies based on the setting in which the study was conducted. ${ }^{12}$ For instance, Hyun Kiyung Kim et al reported that $14.2 \%$ of liver Tx recipients suffered blood stream infection caused by $K$. pneumonia ${ }^{13}$ while the rate of $K$. pneumoniae infections reported by Linares et al was $6.9 \%{ }^{12}$

The concerning issue is the high rate of mortality among patients following K. pneumoniae infection. It was reported in one study that the mortality rate was $32 \%$ and $78 \%$ among patients with carbapenem-resistant and carbapenem-sensitive K. pneumoniae infections, respectively. ${ }^{14}$

The major concern regarding the outcomes associated with K. pneumoniae infection is the high incidence of Carbapenemresistant $K$. pneumoniae (CRKP) vs Carbapenem-sensitive $\mathrm{K}$. pneumoniae (CSKP). It is estimated that the incidence of infections caused by carbapenem-resistant Enterobacteriaceae (CRE), particularly CRKP, is $6 \%$ to $12.9 \%$ in liver $\mathrm{Tx}$ recipients. ${ }^{15}$ In this regard, mortality rate related to CRKP infection was $82 \%$ and $35 \%$ in Mouloudi et al ${ }^{16}$ and Pereira et $\mathrm{al}^{17}$ studies, respectively. In one retrospective cohort study in 2012,37\% of infections in liver Tx recipients were related to Klebsiella. CRPK infections occurred in $72 \%$ of patients and the mortality rate was $71 \%$ amongst this population. Most of the deaths occurred within 30 days after CRKP infection. ${ }^{18}$ Also, Lubbert et al reported that mortality rate in liver Tx recipients infected with carbapenemase producing K. pneumoniae (CPKP) was $78 \%$, with $56 \%$ of the related deaths occurring due to sepsis and multi-organ failure. ${ }^{19}$

\section{Risk Factors}

The spread of infections caused by $K$. pneumoniae is a major concern in the hospitalized patients. Based on the available studies, chronic liver diseases, dialysis, cancers, and solid organ Tx are the main risk factors for K. pneumoniae infections. ${ }^{12}$ Considering the conducted studies, the following can be mentioned as important risk factors for CRKP infections in liver TX recipients: Colonization with CRKP, ${ }^{17,20}$ chronic kidney disease (CKD), model for end-stage liver disease (MELD) score more than 20, mechanical ventilation, ${ }^{20,21}$ exposure to cephalosporine/ carbapenem/piperacillin tazobactam, ${ }^{19,21}$ renal replacement therapy, ${ }^{20} \mathrm{HCV}$ recurrence, ${ }^{20}$ and Roux-en-Y biliary choledochojejunostomy. ${ }^{17}$

\section{Clinical Presentation}

Blood stream ${ }^{20}$ and urinary tract ${ }^{18}$ infections are the most common infections caused by $K$. pneumoniae. Also, pneumonia, ${ }^{20}$ as well as tertiary peritonitis and surgical site infections ${ }^{19}$ have been mentioned as other complications of $K$. pneumoniae in liver Tx recipients.

Rana et al conducted a case series study on necrotizing soft tissue infections (NSTIs) caused by K. pneumoniae in liver Tx recipients, and reported that all 6 patients with NSTI expired, 4 of which were Carbapenem-resistant $K$. pneumoniae (CRKP). Diabetes mellitus (DM), prolonged courses of antimicrobial therapy, history of hospitalization before liver Tx, and non-contiguous areas of necrosis were considered to be the predisposing factors. ${ }^{22}$

\section{Prevention And Treatment}

One of the most effective actions to prevent complications caused by klebsiella infections is to identify the related risk factors and try to eliminate or at least control them.

Although antibiotics such as polymyxins, carbapenems, glycylcyclines, aminoglycosides, cephalosporines, fluoroquinolones, monobactam, fosfomycin, tetracyclines, cotrimoxazole, and beta lactam-beta lactamase inhibitors are recognized as treatment options against $K$. pneumoniae, considering the high prevalence and spread of CPKP species, only polymyxins, aminoglycosides, and tigecycline 
have inhibitory activity against CPKP in vitro. ${ }^{23}$ Also, in recent years, ceftazidime/avibactam has been approved for treatment of hospital and ventilator acquired pneumonia, as well as complicated intraabdominal and urinary tract infections caused by K. pneumoniae carbapenemase (KPC) producing $K$. pneumoniae. This drug which is usually administered $2.5 \mathrm{~g}$ every $8 \mathrm{hrs}$ in adults and has intrinsic activity against Enterobacteriaceae-producing KPCs, extended spectrum beta lactamases (ESBLs), OXA, AmpC enzymes but is not effective against class B $\beta$-lactamases (MBL, VIM, NDM). Also, its activity against CRE species is limited to case series. Considering the resistance of some species to this drug and lack of use in liver Tx recipients, further clinical studies are required to evaluate this drug's efficacy in this population. ${ }^{24-27}$

There are several studies with different results, which have evaluated the outcomes of monotherapy or combined therapy in liver Tx patients with $K$. pneumoniae infection.

A total of 17 Tx recipients with CPKP infection were retrospectively studied by Clancy et al, and the results were as follows: 1) antibiotics that were inactive against CPKP in vitro, did not have appropriate activity in patients. 2) Seventy-one percent of patients treated with monotherapy, experienced treatment failure and loss of susceptibility to gentamicin, colistin, and ciprofloxacin was observed in some cases. Thus, the authors believe that combined therapy is preferred over monotherapy in cases with CPKP infection. 3) In this study, the combination of colistin and doripenem was identified as the most successful antibiotic regimen. ${ }^{28}$

Even though there are some evidence on the effect of carbapenem monotherapy on CPKP, particularly on isolates with carbapenem MICs of $\leq 4 \mathrm{mg} / \mathrm{dL}$, combining antibiotic therapy were preferred in most studies. ${ }^{23}$ Also, in Lee et al review it was concluded that monotherapy with polymyxins achieved the highest rate of failure in comparison with combined therapy of polymyxins with other antibiotics. Whereas combination of colistin with tigecycline, colistin with carbapenem and colistin with aminoglycosides provided the highest rate of success in treating CPKP infections, respectively. ${ }^{29}$

For CPKP infection, prolonged infusion of carbapenems, particularly meropenem is a treatment option. Prolonged infusion maximizes the time that antibiotic concentrations remain above the MIC of microorganism, which reduces the chance of treatment failure. ${ }^{30}$ Pharmacokinetic studies have shown that prolonged infusion of $1 \mathrm{~g}$ meropenem during $3 \mathrm{hrs}$ for every $8 \mathrm{hrs}$ was able to retain the concentration above MIC for a longer period in comparison with infusion of a similar dose during half an hour. ${ }^{30}$ Also, it was mentioned that this treatment protocol $(1 \mathrm{~g}$ meropenem $\mathrm{q} 8 \mathrm{~h}$ over $3 \mathrm{~h}$ ) was more effective against CPKP isolates with MIC $\leq 4$ $\mathrm{mg} / \mathrm{dL}$, and for isolates with KPC $>4 \mathrm{mg} / \mathrm{dL}$, high dose prolonged infusion ( $2 \mathrm{~g}$ meropenem q 8 hrs over $3 \mathrm{hrs}$ ) was recommended.

Tigecycline is another effective antibiotic against KPC. Its volume of distribution ( $\mathrm{Vd})$ is $8 \mathrm{~L}$; hence, it has a strong tissue penetration, can enter into skin, gallbladder, bowel, and pulmonary tissue and is FDA approved for skin and intra-abdominal infections, as well as community acquired pneumonia. This drug is not a good choice in the case of blood stream infections due to its low plasma concentrations, and is not FDA approved for this purpose. Long elimination half-life after multiple dosing and the lack of effect of renal impairment, as well as mild hepatic impairment on drug clearance, can be mentioned as other advantages of this drug. The value of tigecycline monotherapy in K. pneumoniae, especially KPC, is under investigation as a result of the FDA warning in 2010 regarding an increase in mortality. ${ }^{31}$

Mouloudi et al assessed the outcomes of treating liver Tx recipients with tigecycline in their own center. Among 109 liver Tx recipients, 10 cases had positive KRCP culture with MICs of $\leq 4 \mathrm{mg} / \mathrm{dL}$ and were candidates to receive $100 \mathrm{mg}$ loading dose of tigecycline and then $50 \mathrm{mg} \mathrm{q} 12 \mathrm{hrs}$ as the maintenance dose, of which, 2 patients received tigecycline as monotherapy, and 9 were given combined therapy with colistin (5 patients) and colistin - gentamicin (4 patients). The results showed that the intensive care unit (ICU) mortality rate and microbiological response rate were $60 \%$ and $70 \%$, respectively. Superinfection with Pseudomonas aeruginosa was observed in 5 patients. It was declared that tigecycline was well tolerated by all patients; however, its adverse effects, particularly hepatotoxicity which is an important issue in liver Tx recipients were not discussed. ${ }^{16}$ Therefore, more studies regarding the use of tigecycline, particularly for treating KPC infection are required to evaluate the risks.

Tigecycline is usually used in combination with cefoperazone-sulbactam, carbapenems, aminoglycosides, and polymixins. In a metanalysis regarding tigecycline monotherapy versus combination therapy for the treatment of hospital-acquired pneumonia, the authors concluded that tigecycline combination therapy is usually used to treat XDR Gram-negative bacilli infections and has lower mortality rate in compared with monotherapy. However, two 
cohort studies showed no significant difference in mortality rate between the tigecycline monotherapy and combination therapy. ${ }^{32}$ Also, in another study, combination therapy with tigecycline has been recommended in severe infections with no other choice but the study could not prove the superiority of combination therapy to monotherapy. ${ }^{33}$ It seems that further studies are required to evaluate the efficacy of tigecycline combination therapy in compared to monotherapy.

Fosfomycin, as one of the phosphonic acid derivatives, possesses antibacterial effects against Gram-positive bacteria and Enterobacteriaceae including Escherichia coli and $K$. pneumoniae. ${ }^{31,34}$ One important issue that should be considered when administering this antibiotic is the emergence of potential resistance during therapy. Thus, some clinicians suggest using Fosfomycin combination therapy to treat Gram-negative bacteria. ${ }^{34}$ Samonis et al evaluated the synergistic effect of Fosfomycin combined with carbapenems, aminoglycosides, colistin, and tigecycline, who concluded that these combinations had appropriate activity against CPKP. However, further clinical studies are required to confirm this result.

In summary, when considering the results of studies on treating $K$. pneumoniae infections in liver $\mathrm{Tx}$ recipients and the spread of CPKP isolates, it seems that combined therapy (mostly polymyxin with carbapenems or aminoglycosides) was superior to most monotherapy. Extended infusion of carbapenems is another therapeutic method. Using tigecycline and Fosfomycin or adding doxycycline and rifampin to antibiotic regimen to treat KPC requires further research.

Also, prophylactic strategies should be implemented by performing polymerase chain reaction (PCR)-based screening upon patient admission in order to identify KPC, isolation of CPKP positive patients in a separate hospital ward, restricting broad-spectrum antibiotics, especially carbapenems, and practicing hand hygiene, particularly by healthcare providers. ${ }^{19}$

\section{Acinetobacter baumannii (A. baumannii) Epidemiology}

Based on Infectious Diseases Society of America (IDSA) studies, A. baumannii has been identified as one of the six resistant pathogens responsible for mortality and morbidity in patients. $^{35}$ This pathogen can cause blood stream, respiratory tract, and surgical site and wound infections. ${ }^{35}$ Some previous studies reported incidence of $A$. baumannii bacteremia in liver $\mathrm{Tx}$ recipients ranging from $0.8 \%$ to $15.9 \%{ }^{36}$ Also, Kim et al in their cohort study showed that
$10.3 \%$ of the studied liver Tx recipients developed blood stream infections caused by $A$. baumannii. ${ }^{13}$

Gao et al reported $A$. baumannii infection in $62.5 \%$ of liver Tx recipients during 2 weeks after Tx and the median time of infection occurrence was 11.5 days. ${ }^{36}$ In this study, multiple culture positive site, intra-abdominal, and lung were the most common sites of infection. ${ }^{36}$ These results were also confirmed in a study by Kim et al in which the most common sites of infection by $A$. baumannii included biliary tract, lung, and peritoneal cavity. ${ }^{6}$

\section{Risk Factors}

According to previous studies, the following risk factors were identified for $A$. baumannii infection in liver Tx recipients:

Hospital length of stay, ${ }^{6,37}$ ICU length of stay $6,37,38$ hemodialysis after $\mathrm{Tx},{ }^{37,38}$ secondary surgery after liver $\mathrm{Tx}^{38}$ End-Stage Liver Disease (MELD) score before liver $\mathrm{Tx}^{6,38}$ having received broad-spectrum antibiotic ${ }^{39}$ particularly previous exposure to carbapenems, ${ }^{37}$ septic shock, ${ }^{39}$ and high age. ${ }^{37,39}$ In Otan et al study, diabetes and graft dysfunction after liver Tx were also considered as risk factors for $A$. baumannii infection and the mortality rate of $A$. baumannii infection was found to be higher in patients with thrombocytopenia. ${ }^{39}$

Also, prolonged cold ischemia, dialysis after liver Tx, liver Tx due to fulminant hepatitis, colonization with carbapenemresistant A. baumannii (CRAB) before Tx, length of ICU stay following Tx, central venous catheter use and previous use of any antibiotic and, specifically, carbapenem were introduced as risk factors for developing CRAB in previous studies. ${ }^{37,40,41}$

\section{Prevention And Treatment}

Various solutions have been proposed to prevent infections caused by $A$. baumannii including limiting the use of mechanical ventilation, removing unnecessary catheter, ${ }^{36}$ daily chlorhexidine bathing, adherence to hand hygiene and contact precautions, and restricting carbapenem usage. ${ }^{42}$

Nowadays, a majority of $A$. baumannii species have become resistant to third and fourth generation cephalosporins, and the reason is their ability to produce ESBL which leads to cephalosporin resistance. ${ }^{42,43}$

Generally, carbapenems are identified as agent of choice to treat $A$. baumannii infections, ${ }^{42}$ but treating it has become difficult due to the occurrence of carbapenemresistant species including multi drug resistant (MDR) and extensively drug resistant (XDR). ${ }^{42-45}$

In Gao et al study, $75 \%$ of isolated species were CRAB and the rate of was highest in patients with pneumonia. ${ }^{36}$ 
In Kim et al study, $82.4 \%$ of $A$. baumannii species and $33.3 \%$ of Acinetobacter Lwoffii species were carbapenem resistant. $^{6}$

Singh et al concluded that liver $\mathrm{Tx}$ recipients with CRAB infection had lower one-year survival than patients who were not infected with CRAB. ${ }^{46}$

Excessive bleeding, delayed allograft function, high rates of reoperation and longer duration of mechanical ventilation are amongst the complications of CRAB infection. $^{47}$

Polymyxins including Colistin are another options to treat A. baumannii infection. ${ }^{42}$ Polymyxins, identified as a potent bactericidal agent against Gram-negative bacteria, cause bacterial cell death via binding to lipid membrane lipopolysaccharide (LPS). ${ }^{48}$ The bactericidal activity of this antibiotic is determined by the ratio of the area under the curve to MIC (AUC/MIC). ${ }^{42}$

Colistin, an active drug, and colistin methanesulfonate (cms), a prodrug of colistin are two different forms of polymyxins. Cms should be converted to colistin to exert its effect. Hence, it is anticipated that patients are exposed to suboptimal concentration for 2 or 3 days before the drug concentration, reach steady state. Solutions to this concern are to use a loading dose or to administer colistin in combination with other antibiotics. ${ }^{42}$

Generally, A. baumannii resistance to polymyxins is low. For instance, in Freire et al study in 2014, 65 liver Tx recipients were evaluated. Polymyxin-resistant $A$. baumannii (PRAB) was isolated from 7 patients, of which 4 developed infection. The mortality rate was $71 \%$ among this population. ${ }^{48}$ In another study in US, $5.3 \%$ of isolated A. baumannii species were resistant to polymixins. ${ }^{35}$

Tigecycline, sulbactam, fosfomycin, and rifampin are other treatment options against A. baumannii. Each of them has some limitations and therapeutic failures. ${ }^{42}$

Based on the studies discussing A. baumannii infection treatment in liver Tx recipients the following points were concluded:

Colistin in combination with carbapenems is one of the standard treatments of XDR-A. baumannii infections. ${ }^{45}$

Gao et al in a 7-year study evaluated the liver Tx recipients who had developed $A$. baumannii infection. They concluded that in CRAB cases, combination of cefoperazone-sulbactam and tigecycline was successful. Also, in immunocompetent patients, from whom sulbactam resistant species were isolated, sulbactam was as effective as imipenem. Generally, monotherapy is associated with the development of resistant species. ${ }^{36}$ Also, treatment failure was observed with monotherapy in another study which was conducted on solid organ transplant (SOT) recipients. ${ }^{36}$ Thus, combination therapy is recommended, especially in the case of MDR/XDR species. The highest rate of success and treatment failure had occurred when combining colistin with carbapenems or tigecycline, respectively. ${ }^{36,45}$ Shields et al suggested that combining doripenem $500 \mathrm{mg}$ every $8 \mathrm{hrs}$ (infused over $4 \mathrm{hrs}$ if possible) with colistin $5 \mathrm{mg} / \mathrm{kg}$ per day to be considered as the regimen of choice for treating XDR $-A$. baumannii in SOT recipients. The also warned against the emergence of $A$. baumannii resistance toward tigecycline due to monotherapy. ${ }^{45}$

\section{Pseudomonas aeruginosa ( $P$. aeruginosa) Epidemiology}

Pseudomonas aeruginosa ( $P$. aeruginosa) is considered as one of the main microorganisms responsible for bacteremia in hospitalized and immunocompromised patients. ${ }^{49}$ It is estimated that 51,000 healthcare-related infections are developed each ear in the USA due to $P$. aeruginosa. ${ }^{50}$

Lee et al reported that early- and late-onset bacteremia after liver $\mathrm{Tx}$ is different regarding the microbiologic spectrum in a way that early-onset Gram-negative bacteremia occurred mostly due to $P$. aeruginosa. This is of value when determining empiric antibiotic therapy in this population. $^{51}$ It was shown that all episodes of infection due to $P$. aeruginosa were developed 2 months after liver Tx. It is explained by the fact that the most immunosuppression occurs in this period. ${ }^{52}$

The most common infectious complication after liver $\mathrm{Tx}$ is bacteremia (nearly one-third of all post-liver Tx infections) which is developed in $25-35 \%$ of liver $\mathrm{Tx}$ recipients. $P$. aeruginosa is one of the main microorganisms responsible for this kind of infection and was isolated from $6.5 \%$ to $12.7 \%$ of liver $\mathrm{Tx}$ patients with bacteremia. ${ }^{53}$ One study on 233 liver Tx recipients showed that $P$. aeruginosa was the third most common pathogen causing bacteremia in this population $(12.7 \%$,) and the mortality rate was $30 \%$ in $P$. aeruginosa bacteremic patients. $^{54}$ The incidence of infections caused by Gramnegative bacteria has dramatically increased in recent years. Oriol et al recorded episodes of blood stream infections, which occurred during the first year after transplant in SOT recipients (including 50 liver Tx recipients) in a ten-year period, from 2007 until 2016. They reported a statistically significant increase in Gram-negative bacilli BSI from $54.1 \%$ to $93.3 \%$, mainly due to $P$. aeruginosa $(2.4 \%$ to $20.4 \%)$ and $K$. pneumoniae $(7.1 \%$ to $26.5 \%) .{ }^{55}$ 


\section{Risk Factors And Mortality}

The information regarding the risk factors of developing P. aeruginosa infections in liver Tx recipients is limited. ${ }^{56}$ Prior transplantation, nosocomial acquisition, and septic shock at onset are introduced as risk factors for developing XDR P. aeruginosa bacteremia. ${ }^{57}$ Also, one study showed that previous transplantation, hospital-acquired blood stream infection, and prior ICU admission were risk factors for MDR P. aeruginosa isolates. ${ }^{58}$

P. aeruginosa infections are concerning issues in liver Tx recipients due to high mortality and morbidity rate, which complicate the treatment course. Previous studies showed that $P$. aeruginosa infections have incidence and mortality rate ranging from $1.9 \%$ to $15.9 \%$ and $0 \%$ to $30 \%$, respectively, in liver Tx patients. ${ }^{51}$

The problem with this microorganism is its ability to become resistant to different classes of antibiotics, making its treatment a great challenge that influences the prognosis and survival of liver Tx patients. ${ }^{59}$ Between $10.3 \%$ and $51.5 \%$ of $P$. aeruginosa species isolated from blood of SOT recipients are reported to be MDR. ${ }^{53}$

In a prospective study, it was shown that extensive drug-resistant (XDR) P. aeruginosa bacteremia was associated with shorter time from $\mathrm{Tx}$ to bacteremia, higher rates of shock and respiratory failure, more frequent ICU admissions, and higher case-fatality rate. In this study, 318 episodes of bacteremia in SOT patients were documented from 2007 to $2013,15 \%$ of which were developed by $P$. aeruginosa. Nearly $61 \%$ of Pseudomonas strains and $10 \%$ of all cases were XDR. The XDR isolates were resistant to antipseudomonal penicillins and cephalosporins, carbapenems, monobactams, fluoroquinolones, fosfomycin, gentamicin, and tobramycin, but sensitive toward colistin and amikacin. $^{59}$

\section{Treatment And Prevention}

Optimal treatment for non-MDR P. aeruginosa infections has not been established yet. It is recommended to initiate the therapy with a combination of antipseudomonal beta lactams (piperacillin/tazobactam, ceftolozane/tazobactam, ceftazidime, cefepime, or a carbapenem) and aminoglycosides for 3-5 days followed by beta-lactam monotherapy. This strategy will decrease the nephrotoxicity in post-transplant patients, in whom renal failure and coadministration of other nephrotoxic drugs are common. ${ }^{60}$ For MDR $P$. aeruginosa, a combination of two or three antibiotics, including beta-lactams, aminoglycosides, polymyxins and a quinolone is recommended for 7 to 10 days. ${ }^{50,61}$ Novel regimens including colistin, doripenem, aminoglycosides, fosfomycin, and rifampicin have shown efficacy in in vitro studies, and small case series, but clinical experience using these combinations is limited. ${ }^{60}$

Sun et al presented a case of liver $\mathrm{Tx}$ recipient with biliary cast syndrome and intractable MDR Pseudomonas bacteremia who did not respond to conventional antibiotic regimens, including piperacillin/tazobactam, ciprofloxacin, tobramycin, and combination of piperacillin/tazobactam, rifampin, and tobramycin. Finally, the patient received a combination of colistimethate $(100 \mathrm{mg}$ every $36 \mathrm{~h}$ ), doripenem $(250 \mathrm{mg}$ every $12 \mathrm{hrs}$ ), and tobramycin (40 mg every other day) for 5 days and the doses were adjusted based on the patient's creatinine clearance of $16 \mathrm{~mL} / \mathrm{min}$. Surprisingly, the blood cultures became sterile after 62 days of bacteremia. The in vitro susceptibility and synergy tests showed that this unique clinical strain of MDR P. aeruginosa was resistant to doripenem, but susceptible to colistin and tobramycin. Among 2-drug combinations, only doripenem plus tobramycin exhibited additive, but not synergistic activity. In contrast, the combination of colistin, doripenem, and tobramycin was rapidly bactericidal and synergistic. ${ }^{53}$

Prolonged or continuous high dose beta-lactam therapy can also be administered in the case of piperacillin-tazobactam, ceftazidime, meropenem, and doripenem. In patients with nosocomial pneumonia caused by $P$. aeruginosa, the combination of aerosolized antibiotics, such as colistin with intravenous antimicrobial therapy (eg, colistin or beta-lactam) can be effective. $^{60}$

In recent years, novel antipseudomonal antibiotics have become available to overcome $P$. aeruginosa antimicrobial mechanisms of resistance. These agents include ceftolozane-tazobactam, ceftazidime-avibactam, imipenem-cilastatin-relebactam. However, the impact of these new agents has not been evaluated, yet. ${ }^{50}$

Due to high mortality and morbidity caused by multidrug-resistant Gram-negative bacteria, recent guidelines have focused on preventing colonization with the aim at reducing the transmission of these species. Regarding MDR- P.aeruginosa, the guidelines strongly recommend hand hygiene, active screening cultures, contact precautions and using isolation rooms for colonized or infected patients. $^{62}$

\section{Escherichia coli (E. coli) Epidemiology}

Escherichia coli (E. coli), a Gram-negative bacteria, is a member of the bacterial family of Enterobacteriaceae, and 
its primary habitat is in the gastrointestinal tract of human and several warm-blooded animals. ${ }^{63}$ This microorganism can cause systemic infection when it leaves its natural habitat. The patients have to receive immunosuppressants after transplant to prevent rejection and it is thought that these medications can affect host-microbial interactions. One study showed that immunosuppressant therapy can change the gut microbiota, leading to overgrowth of indigenous $E$. coli and increased colonization by uropathogenic E. coli. ${ }^{64}$

E. coli is known to cause late-onset infections, usually beyond the second month after liver transplantation. In Lee et al study, the most common pathogen causing lateonset Gram-negative bacteremia was E. coli. ${ }^{51}$ Also, E. coli was the most frequent bacteria in liver Tx recipients who survived for more than 1-year post-transplant. ${ }^{65}$

As it was mentioned, blood stream infections in liver Tx recipients are often caused by Gram-negative bacilli such as E. coli, K. pneumoniae, and P. aeruginosa. Also, $E$. coli is one of the main pathogens responsible for surgical site infections in this population. ${ }^{4}$

\section{Risk Factors And Mortality}

A 17-year study of blood stream infections caused by E. coli after liver transplantation was conducted in People's Republic of China, and E. coli was the most common amongst patients with blood stream infections, which was associated with an increase in mortality 15 days after infection development. Cholangioenterostomy and ductal complications were identified as risk factors for E. coli bacteremia. Carbapenem and piperacillin-tazobactam were the most consistently active antibiotics against $E$. coli, and the resistance rate to other agents was high. ${ }^{66}$ Bert et al evaluated 704 patients who underwent transplantation in a 10 -year period. The blood cultures showed that the most frequent pathogens were Enterobacteriaceae members (41\%) and the most frequent species was E. coli. ${ }^{67}$

\section{Treatment}

Skin or soft tissue infections have rarely been reported in liver Tx recipients, but they are serious and can even occur in patients with a functional graft. E. coli is the most common Gram-negative bacteria involved in these infections. Empiric therapy against Gram-positive cocci and Gram-negative bacilli should be promptly initiated. ${ }^{68,69}$

Several treatment options exist for severe infections developed by susceptible $E$. coli, such as penicillins, $\beta$-lactam/ $\beta$-lactamase inhibitors, cephalosporins, monobactams, carbapenems, fluoroquinolones, aminoglycosides, and trimethoprim-sulfamethoxazole (TMP-SMX). However, the evolution of resistant species, including fluoroquinolone resistant, as well as ESBL, plasmid-mediated Amp (pAmpC) and carbapenemase producing E. coli has limited the treatment options. ${ }^{70}$

Carbapenems, including imipenem and meropenem are considered as the drug of choice for ESBL infections. These infections are usually resistant to penicillins, fluoroquinolones, TMP-SMX and some aminoglycosides. The risk of clinical failure is higher with cefepime and piperacillin-tazobactam. Hence, these drugs should be used as alternative in patients who are not severely ill. ${ }^{61,70}$

Infections caused by MDR Gram-negative bacteria, including CRE are concerning challenges among solid organ transplantation recipients, which can lead to high mortality rates and graft dysfunction. Recently, occurrence of carbapenemase-producing Enterobacteriaceae (CPE) has emerged in immunosuppressed SOT recipients, which is even more vulnerable than CRE. In a study evaluating CPE acquisition in SOT recipients, KPC-producing E. coli was isolated in $17(3.0 \%)$ patients. The results showed that interspecies spread of carbapenemase genes between carbapenem-resistant $K$. pneumoniae and $E$. coli in a single recipient via mobile genetic cassettes can occur, due to coexistence of these pathogens in colon, especially in SOT recipients with life-long immunosuppressive therapy. ${ }^{71}$

Colistin, tigecyline, fosfomycin, and in some cases, aminoglycosides (gentamycin, amikacin) are against CPE and are administered as combination regimens for treating infections caused by these species. Some studies have recommended that high dose carbapenems should be part of regimens, preferably as prolonged infusions. However, it has been suggested to use monotherapy for uncomplicated urinary tract infections due to complications associated with combination therapy. ${ }^{4,70}$

The marked increase in E. coli non-susceptible to fluoroquinolones is also of particular concern. Hauck et al evaluated fluoroquinolone (ciprofloxacin and levofloxacin) non-susceptibility trends in E. coli species isolated from blood and urine cultures of patients over a 16-year period. They concluded that the annual rate of fluoroquinolone non-susceptibility in $E$. coli increased across the study period 2000-2015 for both blood and urine isolates. ${ }^{72}$

As mentioned before, polymyxins are identified as the last-resort treatment MDR Gram-negative bacteria, including E. coli. Regrettably, the spread of polymyxin resistance is on the rise amongst these microorganisms. In an 
Table I A Summary Of Epidemiology, Risk Factors, Clinical Presentation, Prevention And Treatments Of The Most Common GramNegative Bacteria In Liver Transplant Recipients

\begin{tabular}{|c|c|c|c|c|}
\hline Epidemiology & Risk Factors & $\begin{array}{l}\text { Clinical } \\
\text { Presentation }\end{array}$ & Treatment & Prevention \\
\hline \multicolumn{5}{|c|}{ Klebsiella pneumoniae (K. pneumoniae) } \\
\hline $\begin{array}{l}\text {-The incidence } \\
\text { of infections by } \\
\text { CRE }^{\mathrm{a}} \text {, } \\
\text { particularly } \\
\text { CRKP }^{\mathrm{b}} \text { in liver } \\
\mathrm{Tx}^{\mathrm{C}} \text { recipients: } \\
6 \% \text { to } 12.9 \% \\
- \text { The incidence } \\
\text { of ESBL } \\
\text { infections in liver } \\
\text { Tx recipients: } \\
5.5-7 \%{ }^{\mathrm{e}}\end{array}$ & $\begin{array}{l}\text {-Risk factors for K. pneumoniae } \\
\text { infections: } \\
\text { chronic liver diseases, dialysis, } \\
\text { cancers and solid organ Tx } \\
\text {-Risk factors for CRKP infections in } \\
\text { liver TX recipients: } \\
\text { Colonization with CRKP, CKD } \\
\text { MELD } \\
\text { mechanical ventilation, exposure } \\
\text { to cephalosporine/carbapenem/ } \\
\text { piperacillin tazobactam renal, } \\
\text { replacement therapy, HCV } \\
\text { recurrence, and Roux-en-Y biliary } \\
\text { choledochojejunostomy. }\end{array}$ & $\begin{array}{l}\text { Blood stream } \\
\text { infection, UTI } \\
\text { pneumonia, } \\
\text { tertiary peritonitis } \\
\text { and surgical site } \\
\text { infections }\end{array}$ & $\begin{array}{l}\text {-K. pneumoniae: polymyxins, } \\
\text { carbapenems, glycylcyclines, } \\
\text { aminoglycosides, } \\
\text { cephalosporines, } \\
\text { fluoroquinolones, monobactam, } \\
\text { fosfomycin, tetracyclines, } \\
\text { cotrimoxazole, and beta lactam- } \\
\text { beta lactamase inhibitors } \\
\text {-CPKP: polymyxins, } \\
\text { aminoglycosides, and tigecycline }\end{array}$ & $\begin{array}{l}\text { PCR'-based screening upon } \\
\text { patient admission in order to } \\
\text { identify KPC; isolation of CPKP } \\
\text { positive patients in a separate } \\
\text { hospital ward; restricting broad- } \\
\text { spectrum antibiotic especially } \\
\text { carbapenems; practicing hand } \\
\text { hygiene particularly by healthcare } \\
\text { providers. }\end{array}$ \\
\hline \multicolumn{5}{|c|}{ Acinetobacter baumannii (A. baumannii) } \\
\hline $\begin{array}{l}\text { The Incidence of } \\
\text { A. baumannii } \\
\text { bacteremia in } \\
\text { liver Tx } \\
\text { recipients: } 0.8 \text { to } \\
15.9 \%\end{array}$ & $\begin{array}{l}\text { Hospital length of stay, ICU } \\
\text { length of stay, hemodialysis after } \\
\text { Tx, secondary surgery after liver } \\
\text { Tx, MELD score before liver Tx, } \\
\text { having received broad-spectrum } \\
\text { antibiotic particularly previous } \\
\text { exposure to carbapenems, septic } \\
\text { shock, high age, diabetes graft } \\
\text { dysfunction after liver Tx }\end{array}$ & $\begin{array}{l}\text { blood stream, } \\
\text { respiratory tract, } \\
\text { surgical site and } \\
\text { wound infections }\end{array}$ & $\begin{array}{l}\text { Carbapenems, Polymyxins, } \\
\text { Tigecycline, sulbactam, fosfomycin } \\
\text { and rifampin }\end{array}$ & $\begin{array}{l}\text { Limiting use of mechanical } \\
\text { ventilation, removing unnecessary } \\
\text { catheter, daily chlorhexidine } \\
\text { bathing, adherence to hand } \\
\text { hygiene and contact precautions, } \\
\text { and restricting carbapenem usage. }\end{array}$ \\
\hline \multicolumn{5}{|c|}{ Pseudomonas aeruginosa (P. aeruginosa) } \\
\hline $\begin{array}{l}\text {-The incidence } \\
\text { of infections by } \\
P . \text { aeruginosa in } \\
\text { liver Tx patients: } \\
1.9 \% \text { to } 15.9 \% \\
\text {-The Incidence } \\
\text { of } P \text {. aeruginosa } \\
\text { bacteremia in } \\
\text { liver Tx } \\
\text { recipients: } 6.5- \\
12.7 \%\end{array}$ & $\begin{array}{l}\text { Prior transplantation, nosocomial } \\
\text { acquisition, septic shock, previous } \\
\text { transplantation, hospital-acquired } \\
\text { blood stream infection, and prior } \\
\text { ICU admission }\end{array}$ & $\begin{array}{l}\text { Blood stream } \\
\text { infections, } \\
\text { pneumonia and } \\
\text { intraabdominal } \\
\text { infections }\end{array}$ & $\begin{array}{l}\text { Antipseudomonal beta lactams } \\
\text { (piperacillin/tazobactam, } \\
\text { ceftolozane/tazobactam, } \\
\text { ceftazidime, cefepime, or a } \\
\text { carbapenem), aminoglycosides, } \\
\text { polymyxins, quinolone } \\
\text { doripenem, fosfomycin and } \\
\text { rifampicin }\end{array}$ & $\begin{array}{l}\text { Hand hygiene, active screening } \\
\text { cultures, contact precautions and } \\
\text { using isolation rooms for } \\
\text { colonized or infected patients. }\end{array}$ \\
\hline \multicolumn{5}{|c|}{ Escherichia coli (E. coli) } \\
\hline $\begin{array}{l}\text { The incidence of } \\
\text { ESBL infection: } \\
5.5-7 \%{ }^{\mathrm{e}}\end{array}$ & $\begin{array}{l}\text { Cholangioenterostomy and ductal } \\
\text { complications }\end{array}$ & $\begin{array}{l}\text { Blood stream } \\
\text { infections, surgical } \\
\text { site infections, } \\
\text { UTI, skin and soft } \\
\text { tissue infection }\end{array}$ & $\begin{array}{l}\text {-Susceptible } E \text {. coli: penicillins, } \beta \text { - } \\
\text { lactam/ } \beta \text {-lactamase inhibitors, } \\
\text { cephalosporins, monobactams, } \\
\text { carbapenems, fluoroquinolones, } \\
\text { aminoglycosides, and TMP-SMX' } \\
\text {-ESBL and CRE species: Colistin, } \\
\text { tigecyline, fosfomycin, and in } \\
\text { some cases, aminoglycosides }\end{array}$ & $\begin{array}{l}\text {-ESBL producing E. coli: contact } \\
\text { precautions and hand hygiene } \\
\text {-Carbapenem resistant E. coli: } \\
\text { educating healthcare workers, } \\
\text { contact precautions, patient and } \\
\text { staff cohorting, chlorhexidine } \\
\text { bathing, targeted screening of } \\
\text { contacts and active surveillance, } \\
\text { optimization of hand hygiene, } \\
\text { environmental cleaning, decreased } \\
\text { use of indwelling devices, the } \\
\text { application of antimicrobial } \\
\text { stewardship principles, and } \\
\text { interfacility communication }\end{array}$ \\
\hline
\end{tabular}

Notes: ${ }^{a}$ Carbapenem-resistant Enterobacteriaceae; ${ }^{b}$ Carbapenemase producing $K$. pneumoniae; ${ }^{\mathrm{c}}$ Transplant; ${ }^{\mathrm{d}}$ Extended spectrum beta lactamase; ${ }^{\mathrm{e}}$ The most commonly isolated ESBL-producing species are Klebsiella pneumoniae and Escherichia coli; ' Chronic kidney disease; gModel for end-stage liver disease; 'Uninary tract infection; 'Polymerase chain reaction; 'klebsiella pneumonia carbapenemase; ${ }^{\mathrm{k}}$ Intensive care unit; 'Trimethoprim/sulfamethoxazole. 
American study, E. coli harboring mcr-1, a plasmid-associated gene for polymyxin resistance was detected in a group of liver transplant recipients. ${ }^{73}$

Contact precautions and hand hygiene have been suggested to prevent ESBL producing E. coli. Also, educating healthcare workers, contact precautions, patient and staff cohorting, chlorhexidine bathing, targeted screening of contacts and active surveillance, optimization of hand hygiene, environmental cleaning, decreased use of indwelling devices, the application of antimicrobial stewardship principles, and interfacility communication are preventive strategies against CRE including carbapenem-resistant $E$ coli. ${ }^{74}$

\section{Conclusion}

The incidence of infections due to MDR-GNB in SOT recipients, including liver Tx patients is on the rise and the rate varies based on the center in which the Tx has been performed. The most common MDR-GNB in liver Tx recipients include $A$. baumannii, E. coli, $P$. aeruginosa and $K$. pneumoniae. These patients are more prone to MDR-GNB infections due to their prolonged hospital stay, as well as receiving immunosuppressive agents and broad-spectrum antibiotics. Infections caused by MDR-GNB are associated with poor prognosis, decreased quality of life and survival, as well as increased mortality and morbidity in this population. Thus, special preventive and treatment strategies should be implemented in liver Tx recipients. The most frequent antibiotics recommended for the treatment of MDR-GNB 9nclude carbapenems (ie, meropenem), colistin, fosfomycin, tigecycline, aminoglycosides and antipseudomonal beta lactams (Table 1). ${ }^{15,72}$

\section{Acknowledgment}

The authors wish to thank Mr H. Argasi at the Research Consultation Center (RCC) of Shiraz University of Medical Sciences for his invaluable assistance in editing this manuscript.

\section{Disclosure}

The authors report no conflicts of interest in this work.

\section{References}

1. Black CK, Termanini KM, Aguirre O, Hawksworth JS, Sosin M. Solid organ transplantation in the 21(st) century. Ann Transl Med. 2018;6 (20):409. doi:10.21037/atm.2018.09.68

2. Wadström J, Ericzon B-G, Halloran PF, et al. Advancing transplantation: new questions, new possibilities in kidney and liver transplantation. Transplantation. 2017;101(2):S1-S42. doi:10.1097/TP.000000000000 1563
3. Lim S, Kim EJ, Lee TB, et al. Predictors of postoperative infectious complications in liver transplant recipients: experience of 185 consecutive cases. Korean J Intern Med. 2018;33(4):798-806. doi:10.3904/ kjim.2017.230

4. Righi E. Management of bacterial and fungal infections in end stage liver disease and liver transplantation: current options and future directions. World J Gastroenterol. 2018;24(38):4311-4329. doi:10.3748/wjg. v24.i38.4311

5. Li C, Wen T-F, Mi K, Wang C, Yan L-N, Li B. Analysis of infections in the first 3-month after living donor liver transplantation. World $J$ Gastroenterol. 2012;18(16):1975. doi:10.3748/wjg.v18.i16.1975

6. Kim YJ, Yoon JH, Kim SI, et al. High mortality associated with Acinetobacter species infection in liver transplant patients. Transplant Proc. 2011;43(6):2397-2399. doi:10.1016/j.transproceed.2011.06.011

7. Gagliotti C, Morsillo F, Moro ML, et al. Infections in liver and lung transplant recipients: a national prospective cohort. Eur $J$ Clin Microbiol Infect Dis. 2018;37(3):399-407. doi:10.1007/s10096-0183183-0

8. Qiao B, Wu J, Wan Q, Zhang S, Ye Q. Factors influencing mortality in abdominal solid organ transplant recipients with multidrug-resistant gram-negative bacteremia. BMC Infect Dis. 2017;17(1):171. doi:10.1186/s12879-017-2276-1

9. Cervera C, van Delden C, Gavalda J, Welte T, Akova M, Carratala J. Multidrug-resistant bacteria in solid organ transplant recipients. Clin Microbiol Infect. 2014;20 Suppl 7:49-73. doi:10.1111/1469-0691.12687

10. Ferrarese A, Zanetto A, Becchetti C, et al. Management of bacterial infection in the liver transplant candidate. World J Hepatol. 2018;10 (2):222-230. doi:10.4254/wjh.v10.i2.222

11. Song SH, Li XX, Wan QQ, Ye QF. Risk factors for mortality in liver transplant recipients with ESKAPE infection. Transplant Proc. 2014;46(10):3560-3563. doi:10.1016/j.transproceed.2014.08.049

12. Linares L, Cervera C, Hoyo I, et al. Klebsiella pneumoniae infection in solid organ transplant recipients: epidemiology and antibiotic resistance. Transplant Proc. 2010;42(8):2941-2943. doi:10.1016/j. transproceed.2010.07.080

13. Kim HK, Park YK, Wang HJ, et al. Epidemiology and clinical features of post-transplant bloodstream infection: an analysis of 222 consecutive liver transplant recipients. Infect Chemother. 2013;45 (3):315-324. doi:10.3947/ic.2013.45.3.315

14. Lübbert C, Rodloff AC, Laudi S, et al. Lessons learned from excess mortality associated with Klebsiella pneumoniae carbapenemase 2producing $\mathrm{K}$. pneumoniae in liver transplant recipients. Liver Transplant. 2014;20(6):736-738. doi:10.1002/lt.v20.6

15. Aguado JM, Silva JT, Fernandez-Ruiz M, et al. Management of multidrug resistant Gram-negative bacilli infections in solid organ transplant recipients: SET/GESITRA-SEIMC/REIPI recommendations. Transplant Rev (Orlando). 2018;32(1):36-57. doi:10.1016/j.trre.2017.07.001

16. Mouloudi E, Massa E, Piperidou M, et al. Tigecycline for treatment of carbapenem-resistant Klebsiella pneumoniae infections after liver transplantation in the intensive care unit: a 3-year study. Transplant Proc. 2014;46(9):3219-3221. doi:10.1016/j.transproceed.2014.09.160

17. Pereira MR, Scully BF, Pouch SM, et al. Risk factors and outcomes of carbapenem-resistant Klebsiella pneumoniae infections in liver transplant recipients. Liver Transplant. 2015;21(12):1511-1519. doi:10.1002/lt.24207

18. Kalpoe JS, Sonnenberg E, Factor SH, et al. Mortality associated with carbapenem-resistant Klebsiella pneumoniae infections in liver transplant recipients. Liver Transplant. 2012;18(4):468-474. doi:10.1002/1t.23374

19. Lubbert C, Becker-Rux D, Rodloff AC, et al. Colonization of liver transplant recipients with KPC-producing Klebsiella pneumoniae is associated with high infection rates and excess mortality: a case-control analysis. Infection. 2014;42(2):309-316. doi:10.1007/s15010-013-0547-3

20. Giannella M, Bartoletti M, Morelli MC, et al. Risk factors for infection with carbapenem-resistant Klebsiella pneumoniae after liver transplantation: the importance of pre- and posttransplant colonization. Am J Transplant. 2015;15(6):1708-1715. doi:10.1111/ajt.13136 
21. Patel G, Huprikar S, Factor SH, Jenkins SG, Calfee DP. Outcomes of carbapenem-resistant Klebsiella pneumoniae infection and the impact of antimicrobial and adjunctive therapies. Infect Control Hosp Epidemiol. 2008;29(12):1099-1106. doi:10.1086/592412

22. Rana MM, Sturdevant M, Patel G, Huprikar S. Klebsiella necrotizing soft tissue infections in liver transplant recipients: a case series. Transpl Infect Dis. 2013;15(4):E157-E163. doi:10.1111/tid.2013.15.issue-4

23. Bergamasco MD, Barroso Barbosa M, de Oliveira Garcia D, et al. Infection with Klebsiella pneumoniae carbapenemase (KPC)-producing K. pneumoniae in solid organ transplantation. Transpl Infect Dis. 2012;14(2):198-205. doi:10.1111/j.1399-3062.2011.00688.x

24. Bassetti M, Giacobbe DR, Giamarellou H, et al. Management of KPC-producing Klebsiella pneumoniae infections. Clin Microbiol Infect. 2018;24(2):133-144. doi:10.1016/j.cmi.2017.08.030

25. Shields RK, Potoski BA, Haidar G, et al. Clinical outcomes, drug toxicity, and emergence of ceftazidime-avibactam resistance among patients treated for carbapenem-resistant enterobacteriaceae infections. Clin Infect Dis. 2016;63(12):1615-1618. doi:10.1093/cid/ciw636

26. Tumbarello M, Trecarichi EM, Corona A, et al. Efficacy of ceftazidime-avibactam salvage therapy in patients with infections caused by klebsiella pneumoniae carbapenemase-producing K. pneumoniae. Clin Infect Dis. 2019;68(3):355-364. doi:10.1093/cid/ciy492

27. Athans V, Neuner EA, Hassouna H, et al. Meropenem-vaborbactam as salvage therapy for ceftazidime-avibactam-resistant klebsiella pneumoniae bacteremia and abscess in a liver transplant recipient. Antimicrob Agents Chemother. 2019;63(1). doi:10.1128/AAC.00779-19

28. Clancy CJ, Chen L, Shields RK, et al. Epidemiology and molecular characterization of bacteremia due to carbapenem-resistant Klebsiella pneumoniae in transplant recipients. Am J Transplant. 2013;13 (10):2619-2633. doi:10.1111/ajt.12424

29. Lee GC, Burgess DS. Treatment of Klebsiella pneumoniae carbapenemase (KPC) infections: a review of published case series and case reports. Ann Clin Microbiol Antimicrob. 2012;11:32. doi:10.1186/ 1476-0711-11-32

30. Daikos GL, Markogiannakis A. Carbapenemase-producing Klebsiella pneumoniae: (when) might we still consider treating with carbapenems? Clin Microbiol Infect. 2011;17(8):1135-1141. doi:10.1111/ j.1469-0691.2011.03553.x

31. van Duin D, Kaye KS, Neuner EA, Bonomo RA. Carbapenemresistant Enterobacteriaceae: a review of treatment and outcomes. Diagn Microbiol Infect Dis. 2013;75(2):115-120. doi:10.1016/j. diagmicrobio.2012.11.009

32. Bai XR, Liu JM, Jiang DC, Yan SY. Efficacy and safety of tigecycline monotherapy versus combination therapy for the treatment of hospitalacquired pneumonia (HAP): a meta-analysis of cohort studies. $J$ Chemother. 2018;30(3):172-178. doi:10.1080/1120009X.2018.1425279

33. Cai Y, Bai N, Liu X, Liang B, Wang J, Wang R. Tigecycline: alone or in combination? Infect Dis (Lond). 2016;48(7):491-502. doi:10.3109/ 23744235.2016.1155735

34. Samonis G, Maraki S, Karageorgopoulos DE, Vouloumanou EK, Falagas ME. Synergy of fosfomycin with carbapenems, colistin, netilmicin, and tigecycline against multidrug-resistant Klebsiella pneumoniae, Escherichia coli, and Pseudomonas aeruginosa clinical isolates. Eur J Clin Microbiol Infect Dis. 2012;31(5):695-701. doi:10.1007/s10096-011-1360-5

35. Qureshi ZA, Hittle LE, O'Hara JA, et al. Colistin-resistant Acinetobacter baumannii: beyond carbapenem resistance. Clin Infect Dis. 2015;60(9):1295-1303. doi:10.1093/cid/civ048

36. Gao F, Ye Q, Wan Q, Liu S, Zhou J. Distribution and resistance of pathogens in liver transplant recipients with Acinetobacter baumannii infection. Ther Clin Risk Manag. 2015;11:501-505. doi:10.2147/ TCRM.S82251

37. de Gouvea EF, Martins IS, Halpern M, et al. The influence of carbapenem resistance on mortality in solid organ transplant recipients with Acinetobacter baumannii infection. BMC Infect Dis. 2012;12:351. doi:10.1186/1471-2334-12-351
38. Hsieh CE, Chen YL, Lin PY, et al. Liver transplantation in patients infected with gram-negative bacteria: non-Acinetobacter baumannii and Acinetobacter baumannii. Transplant Proc. 2013;45(1):225-230. doi:10.1016/j.transproceed.2012.09.114

39. Otan E, Aydin C, Usta S, Kutluturk K, Kayaalp C, Yilmaz S. Acinetobacter infection in a liver transplantation intensive care unit. Transplant Proc. 2013;45(3):998-1000. doi:10.1016/j.transproceed.2013. 02.077

40. Freire MP, Pierrotti LC, Oshiro IC, et al. Carbapenem-resistant Acinetobacter baumannii acquired before liver transplantation: impact on recipient outcomes. Liver Transplant. 2016;22(5):615626. doi:10.1002/1t.24389

41. Kim YJ, Kim SI, Lee YD, et al. Carbapenem-resistant acinetobacter baumannii bacteremia in liver transplant recipients. Transplant Proc. 2018;50(4):1132-1135. doi:10.1016/j.transproceed.2018.01.043

42. Doi Y, Murray GL, Peleg AY. Acinetobacter baumannii: evolution of antimicrobial resistance-treatment options. Semin Respir Crit Care Med. 2015;36(1):85-98. doi:10.1055/s-00000075

43. Anvarinejad M, Japoni A, Davarpanah MA, Mahmudi H, Mammina C, Vazin A. Phenotypic and molecular epidemiology of acinetobacter calcoaceticus baumannii complex strains spread at Nemazee Hospital of Shiraz, Iran. Jundishapur journal of microbiology. 2015;8(6).

44. Reddy P, Zembower TR, Ison MG, Baker TA, Stosor V. Carbapenemresistant Acinetobacter baumannii infections after organ transplantation. Transplant Infect Dis. 2010;12(1):87-93. doi:10.1111/tid.2010.12.issue-1

45. Shields RK, Kwak EJ, Potoski BA, et al. High mortality rates among solid organ transplant recipients infected with extensively drug-resistant Acinetobacter baumannii: using in vitro antibiotic combination testing to identify the combination of a carbapenem and colistin as an effective treatment regimen. Diagn Microbiol Infect Dis. 2011;70 (2):246-252. doi:10.1016/j.diagmicrobio.2010.12.023

46. Singh N, Gayowski T, Rihs JD, Wagener MM, Marino IR. Evolving trends in multiple-antibiotic-resistant bacteria in liver transplant recipients: a longitudinal study of antimicrobial susceptibility patterns. Liver Transplant. 2001;7(1):22-26. doi:10.1053/jlts.2001.20769

47. Patel G, Perez F, Bonomo RA. Carbapenem-resistant Enterobacteriaceae and Acinetobacter baumannii: assessing their impact on organ transplantation. Curr Opin Organ Transplant. 2010;15(6):676-682. doi:10.1097/ MOT.0b013e3283404373

48. Freire MP, Van Der Heijden IM, do Prado GV, et al. Polymyxin use as a risk factor for colonization or infection with polymyxin-resistant Acinetobacter baumannii after liver transplantation. Transplant Infect Dis. 2014;16(3):369-378. doi:10.1111/tid.2014.16.issue-3

49. Korvick JA, Marsh JW, Starzl TE, Yu VL. Pseudomonas aeruginosa bacteremia in patients undergoing liver transplantation: an emerging problem. Surgery. 1991;109(1):62-68.

50. Bassetti M, Vena A, Croxatto A, Righi E, Guery B. How to manage Pseudomonas aeruginosa infections. Drugs Context. 2018;7:212527. doi: $10.7573 / 17404398$

51. Lee SO, Kang SH, Abdel-Massih RC, Brown RA, Razonable RR. Spectrum of early-onset and late-onset bacteremias after liver transplantation: implications for management. Liver Transplant. 2011;17 (6):733-741. doi:10.1002/lt.22296

52. Zhong ZQ, Luo AJ, Wan QQ, Ye QF. Pseudomonas aeruginosa infection among liver transplant recipients: a clinical analysis of 15 cases. Transplant Proc. 2016;48(6):2130-2134. doi:10.1016/j.transproceed. 2016.03.052

53. Sun HY, Shields RK, Cacciarelli TV, Muder RR, Singh N. A novel combination regimen for the treatment of refractory bacteremia due to multidrug-resistant Pseudomonas aeruginosa in a liver transplant recipient. Transplant Infect Dis. 2010;12(6):555-560. doi:10.1111/ j.1399-3062.2010.00543.x

54. Singh N, Wagener MM, Obman A, Cacciarelli TV, de Vera ME, Gayowski T. Bacteremias in liver transplant recipients: shift toward gram-negative bacteria as predominant pathogens. Liver Transplant. 2004;10(7):844-849. doi:10.1002/(ISSN)1527-6473 
55. Oriol I, Sabe N, Simonetti AF, et al. Changing trends in the aetiology, treatment and outcomes of bloodstream infection occurring in the first year after solid organ transplantation: a single-centre prospective cohort study. Transplant Int. 2017;30(9):903-913. doi:10.1111/tri.2017.30.issue-9

56. Liu T, Zhang Y, Wan Q. Pseudomonas aeruginosa bacteremia among liver transplant recipients. Infect Drug Resist. 2018;11:2345-2356. doi:10.2147/IDR.S180283

57. Bodro M, Sabe N, Tubau F, et al. Extensively drug-resistant Pseudomonas aeruginosa bacteremia in solid organ transplant recipients. Transplantation. 2015;99(3):616-622. doi:10.1097/TP.00000000 00000366

58. Johnson LE, D'Agata EM, Paterson DL, et al. Pseudomonas aeruginosa bacteremia over a 10-year period: multidrug resistance and outcomes in transplant recipients. Transpl Infect Dis. 2009;11(3):227-234.

59. Bonomo RA, Szabo D. Mechanisms of multidrug resistance in Acinetobacter species and Pseudomonas aeruginosa. Clin Infect Dis. 2006;43 Suppl 2:S49-S56. doi:10.1086/504477

60. van Duin D, van Delden C. Multidrug-resistant gram-negative bacteria infections in solid organ transplantation. Am J Transplant. 2013;13(Suppl 4):31-41. doi:10.1111/ajt.12096

61. Santoro-Lopes G, de Gouvea EF. Multidrug-resistant bacterial infections after liver transplantation: an ever-growing challenge. World $J$ Gastroenterol. 2014;20(20):6201-6210. doi:10.3748/wjg.v20.i20.6201

62. Tacconelli E, Cataldo MA, Dancer SJ, et al. ESCMID guidelines for the management of the infection control measures to reduce transmission of multidrug-resistant Gram-negative bacteria in hospitalized patients. Clin Microbiol Infect. 2014;20 Suppl 1:1-55. doi:10.1111/ 1469-0691.12427

63. Allocati N, Masulli M, Alexeyev MF, Di Ilio C. Escherichia coli in Europe: an overview. Int J Environ Res Public Health. 2013;10 (12):6235-6254. doi:10.3390/ijerph10126235

64. Tourret J, Willing BP, Dion S, MacPherson J, Denamur E, Finlay BB. Immunosuppressive treatment alters secretion of ileal antimicrobial peptides and gut microbiota, and favors subsequent colonization by uropathogenic Escherichia coli. Transplantation. 2017;101(1):74-82. doi:10.1097/TP.0000000000001492
65. Aberg F, Makisalo H, Hockerstedt K, Isoniemi H. Infectious complications more than 1 year after liver transplantation: a 3-decade nationwide experience. Am J Transplant. 2011;11(2):287-295. doi:10.1111/ajt.2011.11.issue-2

66. Tai Q, He XS, Hu AB, et al. [A 17-year study of bloodstream Escherichia coli infection after liver transplantation: resistance rate, risk factor and mortality]. Zhonghua Yi Xue Za Zhi. 2011;91(42):2977-2980.

67. Bert F, Larroque B, Paugam-Burtz C, et al. Microbial epidemiology and outcome of bloodstream infections in liver transplant recipients: an analysis of 259 episodes. Liver Transplant. 2010;16(3):393-401. doi:10.1002/lt.21991

68. Janny S, Bert F, Dondero F, et al. Fatal Escherichia coli skin and soft tissue infections in liver transplant recipients: report of three cases. Transplant Infect Dis. 2013;15(2):E49-E53. doi:10.1111/tid.2013.15.issue-2

69. Paterson DL, Gruttadauria S, Lauro A, Scott V, Marino IR Spontaneous gram-negative cellulitis in a liver transplant recipient. Infection. 2001;29(6):345-347. doi:10.1007/s15010-001-1112-Z

70. Vila J, Saez-Lopez E, Johnson JR, et al. Escherichia coli: an old friend with new tidings. FEMS Microbiol Rev. 2016;40(4):437-463. doi:10.1093/femsre/fuw005

71. Lee KH, Han SH, Yong D, et al. Acquisition of carbapenemase-producing enterobacteriaceae in solid organ transplantation recipients. Transplant Proc. 2018;50(10):3748-3755. doi:10.1016/j.transproceed.2018.01.058

72. Hauck CG, Chong PP, Miller MB, et al. Increasing rates of fluoroquinolone resistance in Escherichia coli isolated from the blood and urine of patients with hematologic malignancies and stem cell transplant recipients. Pathog Immun. 2016;1(2):234-242. doi:10.20411/pai.v1i2.115

73. Macesic N, Khan S, Giddins MJ, et al. Escherichia coli harboring mcr-1 in a cluster of liver transplant recipients: detection through active surveillance and whole-genome sequencing. Antimicrob Agents Chemother. 2019;63(6). doi:10.1128/AAC.02680-18

74. Pouch SM, Patel G. Multidrug-resistant Gram-negative bacterial infections in solid organ transplant recipients-Guidelines from the American Society of Transplantation Infectious Diseases Community of Practice. Clin Transplant. 2019; 13594.
Infection and Drug Resistance

\section{Publish your work in this journal}

Infection and Drug Resistance is an international, peer-reviewed openaccess journal that focuses on the optimal treatment of infection (bacterial, fungal and viral) and the development and institution of preventive strategies to minimize the development and spread of resistance. The journal is specifically concerned with the epidemiology of

\section{Dovepress}

antibiotic resistance and the mechanisms of resistance development and diffusion in both hospitals and the community. The manuscript management system is completely online and includes a very quick and fair peerreview system, which is all easy to use. Visit http://www.dovepress.com/ testimonials.php to read real quotes from published authors 